\title{
Stool as Appropriate Sample for the Diagnosis of Mycobacterium tuberculosis by Gene Xpert Test
}

\author{
Seble Haile Welday ${ }^{*}$, Andrew Nyerere Kimang'a ${ }^{1}$, Beatrice Muthoni Kabera ${ }^{2}$, \\ Josphine Wahogo Mburu ${ }^{3}$, Christina Mwachari ${ }^{4}$, Elizabeth Mungai ${ }^{5}$, \\ Stanley Mugambi Ndwiga², Joseph Kariuki Mbuthia², Gunturu Revathi6 \\ ${ }^{1}$ Department of Medical Microbiology, Jomo Kenyatta University of Agriculture and Technology, Nairobi, Kenya \\ ${ }^{2}$ Gertrude Children's Hospital, Nairobi, Kenya \\ ${ }^{3}$ National Reference TB Laboratory, Nairobi, Kenya \\ ${ }^{4}$ University of Maryland Program, Nairobi, Kenya \\ ${ }^{5}$ Kiambu District Hospital, Kiambu, Kenya \\ ${ }^{6}$ Department of Pathology, Aga Khan University Hospital, Nairobi, Kenya \\ Email: seblehe161@yahoo.com, ${ }^{*}$ henseb03@gmail.com
}

Received 20 May 2014; revised 20 June 2014; accepted 31 July 2014

Copyright (C) 2014 by authors and Scientific Research Publishing Inc.

This work is licensed under the Creative Commons Attribution International License (CC BY).

http://creativecommons.org/licenses/by/4.0/

cC) (i) Open Access

\section{Abstract}

Background: Diagnosis of pediatric pulmonary tuberculosis (PTB) is a challenge. Symptoms are nonspecific. Young children are unable to expectorate sputum samples; the procedures for obtaining respiratory samples are invasive. Thus Mycobacterium tuberculosis cultures and smears often are not performed. Stool samples were used as an alternative to respiratory samples for the diagnosis of pediatric PTB using stool Xpert MTB/RIF and its sensitivity for detecting the DNA of MTB in stool was determined. Methods: The study was a laboratory-based cross-sectional prospective design. Stool specimen was collected from PTB suspected children ( $<15$ years) attended in Gertrude's Children's Hospital Nairobi and Kiambu District Hospital from September 2013 to March 2014. Stool for Xpert was processed in two ways, direct and prior extraction of DNA using QIAGEN stool DNA extraction kit. Result: A total sample of 91 stool specimen was collected from patients. Of these $53(58.2 \%)$ had sputum ZN smear microscopy. Six $(11.3 \%)$ of them were confirmed smear positive for PTB. Stool Gene Xpert was positive in all the six smear positive children. Four (7.5\%) smear negative patients tested positive by stool Gene Xpert test. This association is significant $(\mathrm{P}=\mathbf{0 . 0 0 0})$. Conclusion: This study reports that Mycobacterium tuberculosis DNA can be detected in stool using Xpert testing with a higher sensitivity. Therefore stool which can easily be obtained is an appropriate alternative sample for the diagnosis of PTB using Xpert assay for children unable to give respiratory samples. Furthermore Xpert turn round time is less than 2 hours.

*Corresponding author.

How to cite this paper: Welday, S.H., Kimang'a, A.N., Kabera, B.M., Mburu, J.W., Mwachari, C., Mungai, E., Ndwiga, S.M., Mbuthia, J.K. and Revathi, G. (2014) Stool as Appropriate Sample for the Diagnosis of Mycobacterium tuberculosis by Gene Xpert Test. Open Journal of Respiratory Diseases, 4, 83-89. http://dx.doi.org/10.4236/ojrd.2014.43012 


\section{Keywords}

\section{Pulmonary Tuberculosis (PTB), Pediatric, Stool, Xpert MTB/RIF, Smear Microscopy, Mycobacterium tuberculosis}

\section{Introduction}

Tuberculosis (TB) is a worldwide pandemic as it is a serious cause of illness and death. In 2011, the infection of the new cases was estimated 8.7 million where 1.4 million people died from it [1]. Millions of people can die over it if control measures are not taken properly [2]. The African region witnessed a sharp rise in the incidence of TB accounting $24 \%$ of the world's cases. Kenya with the large TB disease burden ranked 13th among the 22 high burden countries, where HIV epidemic, poor socio-economic status leading to overcrowded slums, coupled with poor nutrition and limited access to health services have been identified as contributing factors [3].

A child is defined as any individual who is under 15 years of age. Childhood tuberculosis is estimated to constitute about $6 \%$ out of all incident cases, with the majority of cases occurring in high TB burden countries [1]. In Kenya, TB in children accounts for about $10 \%$ of all cases every year [3]. The risk of infection in children depends on the extent of exposure to infectious droplet nuclei. Generally the frequency of childhood TB depends on the intensity of the epidemic, the age structure of the population, the available diagnostic tools and whether contact tracing is routinely undertaken [4].

TB diagnosis in children usually follows discovery of a case in adults, tuberculin skin testing, chest radiograph, and clinical signs and symptoms, however, none of these are fully efficient to diagnose a case [5]. Despite the advances made in diagnostic of adult TB, development in children is lagging behind [6]. The difficulty of establishing a definitive diagnosis of TB in children is due to several reasons. Symptoms are nonspecific that can also be present in other viral and bacterial infections. Consequently, TB is usually hard to recognize based on symptoms alone. The standard diagnostic test for TB requires a sample of sputum which is very difficult to obtain as young children are unable to expectorate sputum. Because good sputa are not accessible, Mycobacterium tuberculosis cultures and smears are often not performed though the disease is in progress. Procedures can be done to obtain samples from a child's lung or stomach but these can be traumatic for the child. It is therefore desirable to develop a non-sputum-based TB diagnostic test for young children that give a fast result. So that children, who are at high risk of dying from TB, can get treatment [7]. Young children tend to swallow sputum when they cough. It is also known that tuberculosis DNA can survive intestinal transit [8]. Therefore, testing stool for tuberculosis DNA from swallowed sputum can diagnose pulmonary tuberculosis and sample collection can easily take place in the field or in clinics.

Diagnosis of pediatric pulmonary tuberculosis (PTB) is a challenge. So this study was conducted to find a solution to the challenges of pediatric TB diagnosis by testing stool sample as an alternative to a respiratory sample in Xpert MTB/RIF assay as well as to determine the sensitivity of Xpert both in direct stool testing method and by prior extraction of stool DNA using QIAGEN stool extraction technique.

\section{Patients and Methods}

The study was a laboratory based cross-sectional prospective design. It was carried out in Gertrude Children's Hospital (private hospital) Nairobi and Kiambu District Hospital (public hospital) in Kiambu County, Kenya. The laboratory analysis was done at the pathology department of the Aga Khan University Hospital, and in the National Tuberculosis Reference Laboratory, Nairobi.

Consecutive children patients (age $<15$ ) referred by their clinicians for TB testing (tuberculin test, CXR, sputum smear and or TB culture) were recruited from September 2013 to March 2014. Consent was sought from their parents or guardians or their legal representatives. Demographic data and history of contact of the children suspected for PTB was obtained using a structured questionnaire. Clinical features and examination results like chest radiography suggestive for PTB, Tuberculin Skin Test and sputum Ziehl-Neelsen (ZN) smear microscopy (were available) were obtained from patients files. The study was not in a position to do all the clinical examinations as well as tests using respiratory samples, due to several limitations. The results were obtained from the hospital's laboratory when available. Plastic disposable pots were used to collect stool samples from patients 
which were stored at $-20^{\circ} \mathrm{C}$ if the testing was going to take longer than 2 hours. Prior to the Xpert testing, stool was processed in two ways.

\subsection{Direct Stool Processing}

$0.15 \mathrm{~g}$ of thawed stool (confirmed by weighing) was picked by a wooden stick commonly used in laboratory processes. It was then placed in $2.4 \mathrm{ml}$ PBS and vortexed briefly before removing the swab. The sample was left undisturbed for $20 \mathrm{~min}$ at room temperature, to allow large particles to sediment before removing 2 aliquots of $1 \mathrm{ml}$ supernatant. One aliquot was tested immediately with Xpert and the other was stored at $4^{\circ} \mathrm{C}$ for later duplicate testing (within 1 week) as needed. One aliquot was centrifuged at $3200 \mathrm{~g}$ for 10 minutes. Xpert testing was then performed as per the manufacturer's instructions, using a 2:1 ratio of Xpert reagent to sample [9].

\subsection{Prior Extraction of DNA}

DNA was isolated from stool using the QIAGEN stool DNA extraction kit as per the manufacturer's instructions [10]. The procedure comprises the following steps:

1) Lysis of stool samples in Buffer ASL;

2) Adsorption of impurities to Inhibit EX;

3) Purification of DNA on QIAamp spins columns.

Then molecular water was added to attain the $2 \mathrm{ml}$ volume needed for the 1:2 ratios (reagent dilution) of the Gene Xpert test.

Ethical clearance for the study was attained from Kenyatta University Ethical Review committee (PKU/ 127/E160) and Gertrude’s Children's Hospital Ethical Review Board.

\section{Result}

A total of 91 children suspected of pulmonary tuberculosis (PTB) were recruited during the study from one private and one public hospital in Kenya. These children were suspected for PTB as they showed 3 or more clinical features in the scoring system that is cough more than two weeks, weight loss, fever or/and night sweat and history of contact [3]. Then they were subjected to tuberculin skin test (TST) and/or chest X-ray (CXR) for respiratory signs suggestive of TB, as well as sputum ZN microscopy (where available) [11]. Stool Gene Xpert was done to all the children suspected of having PTB. The following Figures 1-4 and Table 1 and Table 2 show the demographic data, clinical features observed and the results obtained from the tests done.

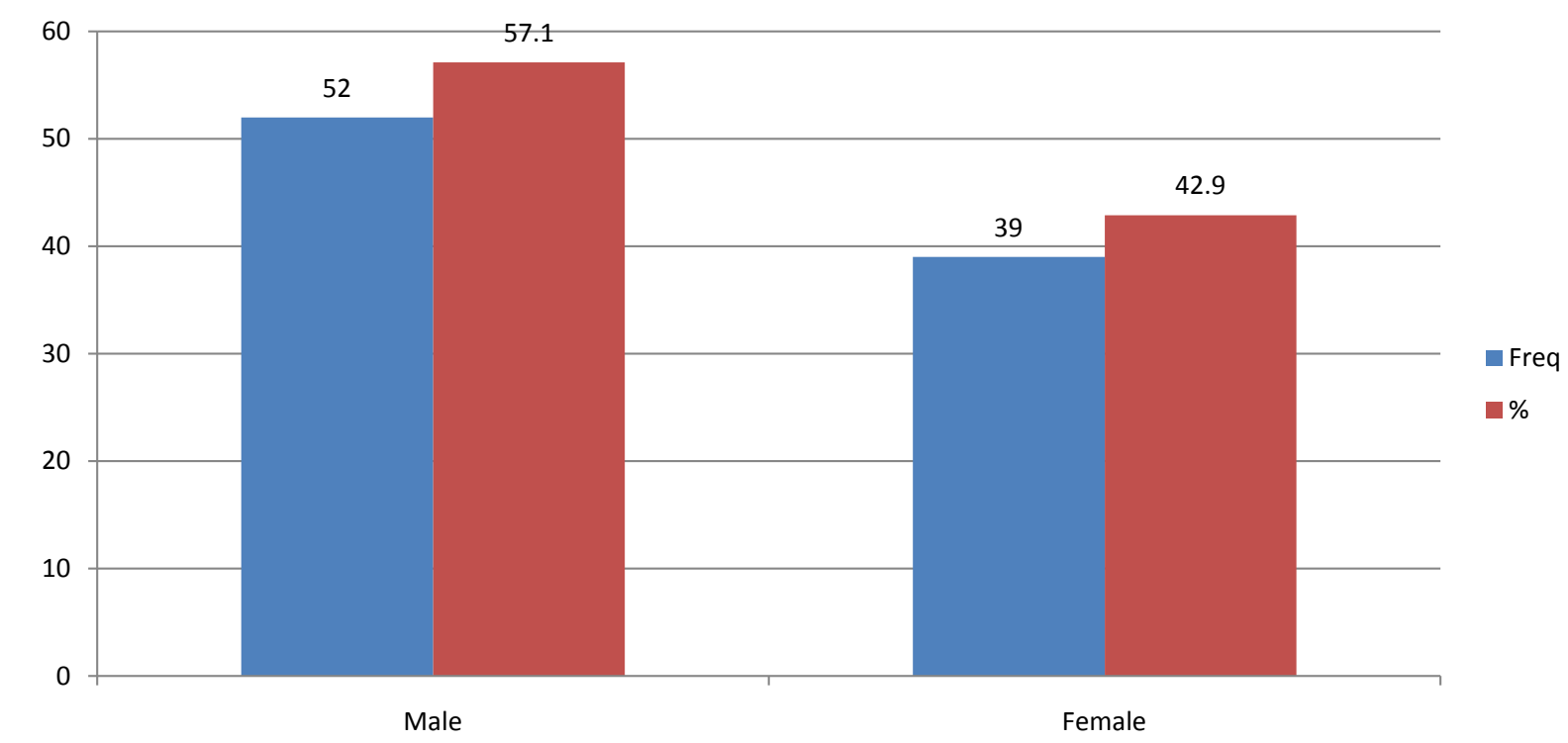

Figure 1. Shows the demographic sex distribution of the children patients in frequency and percentage. The total numbers of children mean age was 4.8 . The minimum age was 1 month while the maximum age was 14 years and 11 months. The most frequent age was 3 years old. 


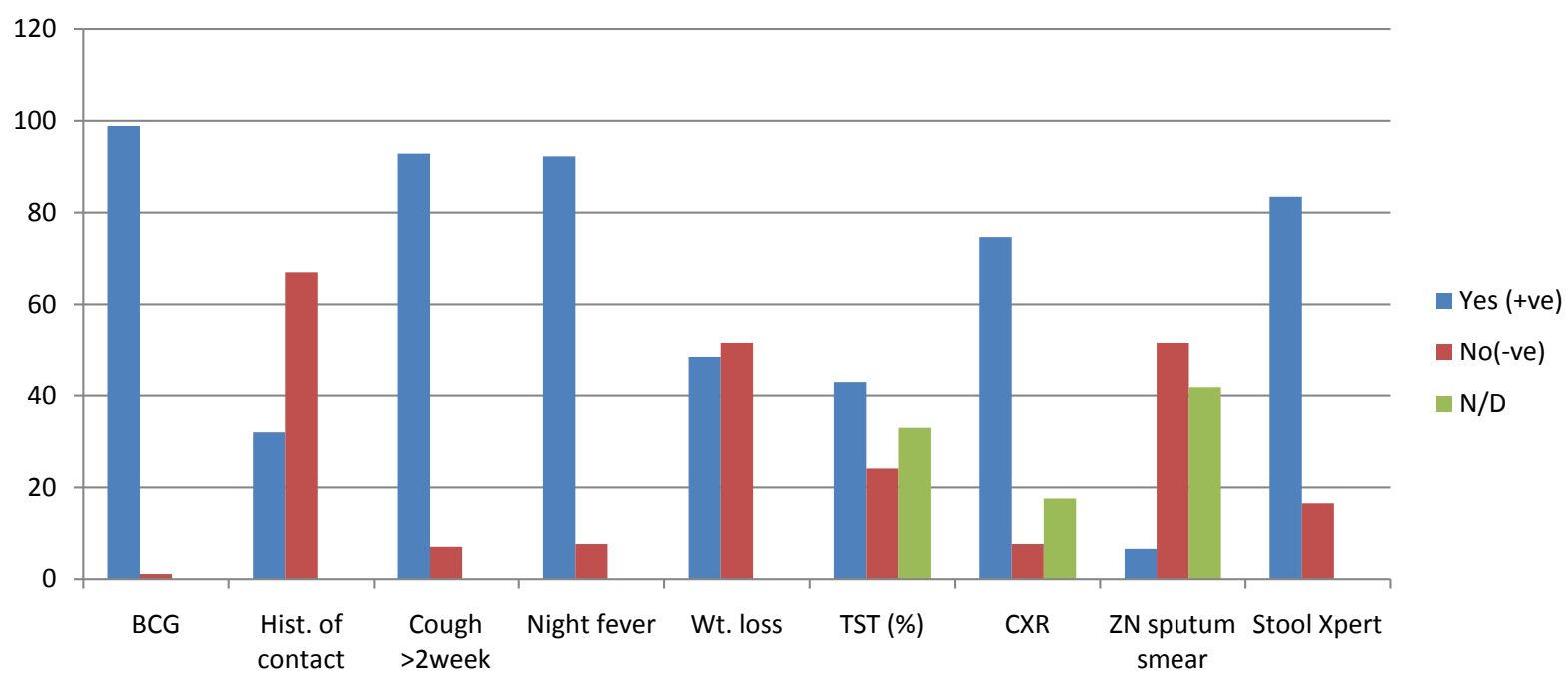

Figure 2. Most common clinical features and tests used for the diagnosis of PTB (in percentage). In this figure Yes (+ve) is positive to clinical features and examinations suggestive for pulmonary tuberculosis; BCG vaccination was done to all except one. No ( - ve) implies the absence of clinical features and examination results suggestive to PTB. N/D stands for tests not done. From this figure it can be observed that these children were showing not all but most of the clinical features and examinations as positive which made them to be suspected for PTB.

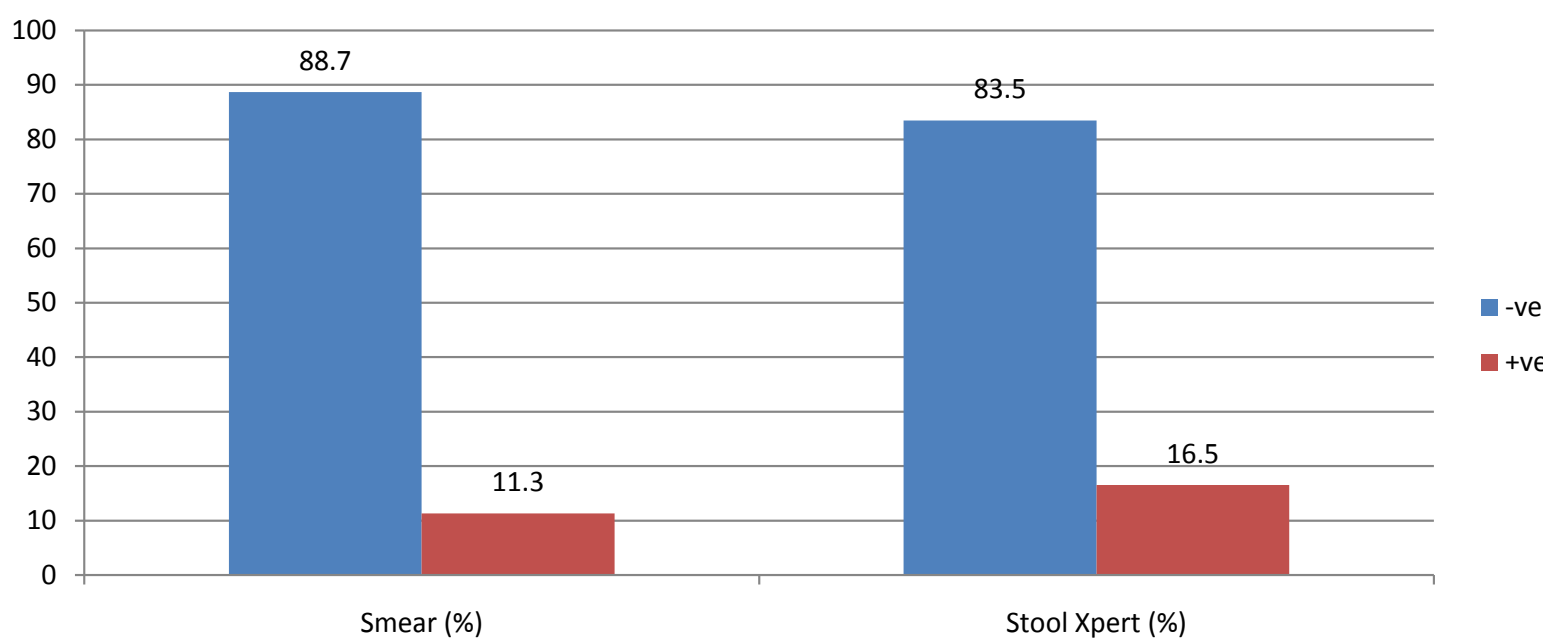

Figure 3. ZN-Sputum smears microscopy vs. stool Gene Xpert tests: this figure shows the percentage of positive and negative results after both ZN smear microscopy (where available) and stool Gene Xpert tests was done for the samples from the 53 children suspected for having PTB. From the 91 children only 53 had ZN microscopy test because of the unavailability of the needed facilities for doing the procedures to obtain the respiratory samples from the children in the public hospital, and some couldn't go through that procedure due to very serious illness rendering them unfit for procedures.

Table 1. Comparison and associations of the results obtained by the sputum ZN smear microscopy (were available) with the results of the stool Gene Xpert assay tests.

\begin{tabular}{ccccc}
\hline & Stool Xpert & Total & P-Value \\
\hline Sputum smear & - ve & + ve & & $\mathbf{0 . 0 0 0}$ \\
-ve & 42 & 5 & 47 & \\
+ve & 0 & 6 & 6 & \\
Total & 42 & 11 & 53 & \\
\hline
\end{tabular}


Table 2. Comparison and associations of the results of Gene Xpert assay tests, obtained by the direct processing of stool with the results of the prior extraction of the DNA using QIAGEN stool DNA extraction kit.

\begin{tabular}{ccccc}
\hline & & Ex. DNA & Total & P-Value \\
\hline Direct & - ve & +ve & & \\
- ve & 76 & 3 & 79 & \\
+ve & 4 & 8 & 12 & \\
Total & 80 & 11 & 91 & \\
\hline
\end{tabular}

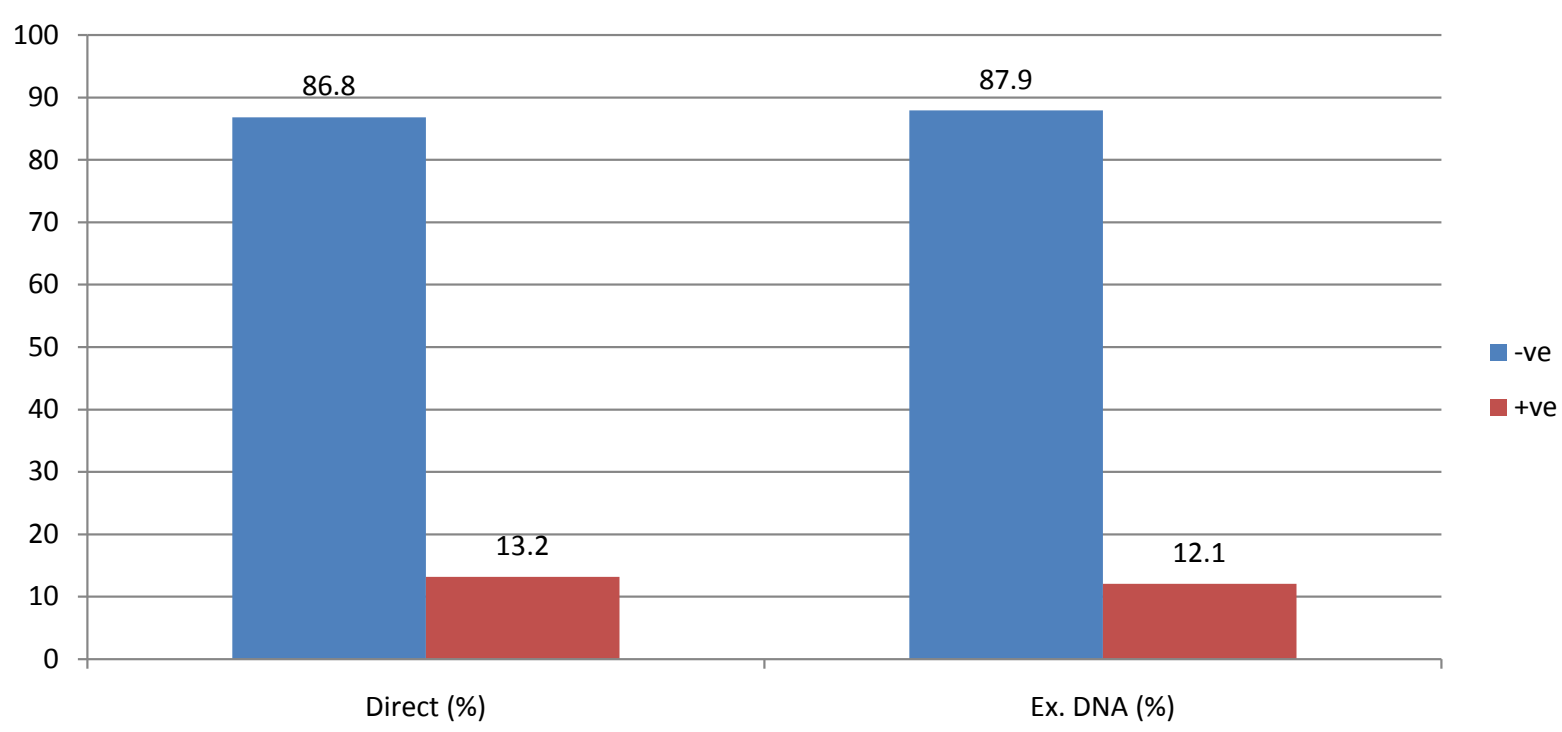

Figure 4. Direct stool Gene Xpert vs after DNA extraction: this figure shows the percentage of positive and negative results for both direct stool and prior extraction of stool DNA. Stool samples from the 91 children suspected of PTB were tested.

\section{Discussion}

Stool which can easily be obtained from clinics and the fields is an appropriate sample for the diagnosis of $M$. tuberculosis in children. Results from this study show that MTB DNA in stool can be detected using Gene Xpert. From the 91 children suspected for pulmonary tuberculosis 53 (58.2\%) were able to do sputum ZN smear microscopy while all of the (91) did stool Xpert test. From the tests done using sputum ZN smear microscopy, direct stool Xpert and prior extracted DNA stool Xpert 6 (11.3\%), 12 (13.2\%) and 11 (12.1\%) were detected as positive respectively $(\mathrm{P}=0.000)$. Stool Xpert showed $100 \%$ sensitivity and $89.36 \%$ specificity without missing any positive from sputum ZN smear microscopy. Compared to direct stool Xpert prior extraction of DNA using QIAGEN stool DNA extraction kit is a long process with $\mathrm{P}=0.001$, this may not be used as routine diagnosis method but can be used as a compliment for further investigation because it helps to detect cases missed in the direct tool Xpert test. Stool PCR would be a rapid, non-invasive, and relatively bio-secure initial test for children with suspected pulmonary tuberculosis [12]. Thus using stool as a sample is a good solution to the invasive procedure for collecting respiratory samples or sputum from children. Pilot study done in South Africa indicated that stool Xpert as a promising test for diagnosis of PTB [9]. Xpert is a portable, highly sensitive, user-friendly and rapid molecular assay with the turnaround time less than 2 hours. Though the issue of affordability and the need of uninterrupted power supply can be some of the drawbacks, in settings where Xpert MTB/RIF is available, stool Xpert testing is a good solution for children of pulmonary tuberculosis with difficulty in producing respiratory samples.

TB diagnosis in children is a challenge [13]. This is because the sputum smear microscopy is not possible for the majority of young children, who usually swallow their sputum. Other methods of obtaining like gastric lavage can be problematic to implement as a routine diagnostic procedures. This means that bacteriological confirmation is usually not possible and that the diagnosis of PTB in children is often presumptive [4]. Therefore as 
observed in the study different tests are done for the diagnosis of childhood PTB along with the other clinical features. All results of the routine tests done in the hospitals like Tuberculin Skin Test (TST), Chest X-Ray (CXR) and sputum ZN smear microscopy (were available) were taken from the patient's filed record. From the 61 (67.0\%) who did Tuberculin Skin Test (TST), 39 (42.9\%) were positive while 22 (24.1\%) were negative. A positive tuberculin test does not necessarily indicate the presence or extent of tuberculosis disease; it only indicates infection and if negative does not necessarily exclude TB infection [14]. The tuberculin test is less likely to be positive in a child with TB if the child also has severe malnutrition, HIV infection or disseminated TB such as TB meningitis [11]. Chest X-Ray (CXR) was done to 75 (82.4\%) children, where 68 (74.7\%) showed changes suggestive for PTB and 7 (7.7 \%) did not. Chest X-ray is required along with clinical symptoms, but often overlaps with other respiratory diseases. However, it can be more specific when the child has been in close contact with a diagnosed case of PTB, especially smear-positive PTB [4].

\section{Conclusion}

Stool sample has the potential of detecting Mycobacterium tuberculosis using Gene Xpert MTB/RIF assay. Introduction of testing stool using Gene Xpert as the routine pulmonary tuberculosis diagnosis in children patients suspected of having PTB could be a solution to the current challenges of getting respiratory samples.

\section{Acknowledgements}

The Authors gratefully thank the institutional support provided by Jomo Kenyatta University of Agriculture and Technology, Gertrude’s Children's Hospital, National Reference Tuberculosis Laboratory, Aga Khan University Hospital pathology department and Kiambu District Hospital. Higher Education Board of Eritrea and African Development Bank are gratefully acknowledged for funding this research. We would also like to thank Dr. Thomas Ngwiri, Prof. M. Mwau, Dr. A. Waititu, Truphena A. Otinyo and N. Kuria for their remarkable assistance.

\section{References}

[1] World Health Organization (2012) Global Tuberculosis Control. World Health Organization, Geneva.

[2] Grange, J.M. and Zumela, A. (2002) The Global Emergency of Tuberclosis: What Is the Cause? Journal of Royal Society for the Promotion of Health, 122, 78-81. http://dx.doi.org/10.1177/146642400212200206

[3] GOK, Ministry of Public Health and Sanitation (2009) DLTLD Guidelines on Management of Leprosy and Tuberculosis. Government Printer, Nairobi.

[4] World Health Organization (2003) Treatment of Tuberculosis Guideline for National Programs. World Health Organization, Geneva.

[5] Khan, E.A. and Starke, J.R. (2006) Diagnosis of Tuberculosis in Children: Increased Need for Better Methods. International Journal of Tuberculosis and Lung Disease, 10, 1091-1097.

[6] Mukherjee, A., Lodha, R. and Kabara, S.K. (2012) Recent Advances in Diagnosis of Tuberculosis. Journal of Pediatric Infectious Diseases, 4, 45-50.

[7] Medicines Sans Frontieres for HIV and Tuberculosis (TB) (2010) Diagnosing Children, a Terrible Neglect. WHO, Geneva.

[8] Cordova, J., Shilho, R., Gilman, R.H., et al. (2010) Evaluation of Molecular Tools for Detection and Drug Susceptibility Testing of Mycobacterium Tuberculosis in Stool Specimens from Patients with Pulmonary Tuberculosis. Journal of Clinical Microbiology, 48, 1820-1826. http://dx.doi.org/10.1128/JCM.01161-09

[9] Nicol, M.P., Spiers, K., Workman, L., Isaacs, W., Munro, J., Black, F., Zemanay, W. and Zar, H.J. (2013) Xpert MTB/RIF Testing of Stool Samples for the Diagnosis of Pulmonary Tuberculosis in Children. Clinical Infectious Disease Oxford Journals, 57, 2-12.

[10] QIAamp (2012) QIAamp DNA Stool Handbook for DNA Purification from Stool Samples. 2nd Edition, New England Biolabs, Ipswich. www.qiagen.com

[11] GOK, Ministry of Public Health and Sanitation (2013) National Guidelines on Management of Tuberculosis in Children. 2nd Edition, Government Printer, Nairobi.

[12] Wolf, H., Mendez, M., Gilman, R.H., Sheen, P., Soto, G., Velarde, A.K., Zimic, M. and Escombe, A.R. (2008) Diagnosis of Pediatric Pulmonary Tuberculosis by Stool PCR. America Journal of Tropical Medicine Hygiene, 79, 893-898. 
[13] Triasih, R. and Graham, S.M. (2011) Limitations of the Indonesian Pediatric Tuberculosis Scoring System in the Context of Child Contact Investigation. Paediatrica Indonesiana, 51, 332-337.

[14] Fjällbrant, H. (2008) BCG Vaccination and the Tuberculin Skin Test in a Country with Low Prevalence of Tuberculosis. Geson Hylte Tryck, Sweden. 
Scientific Research Publishing (SCIRP) is one of the largest Open Access journal publishers. It is currently publishing more than 200 open access, online, peer-reviewed journals covering a wide range of academic disciplines. SCIRP serves the worldwide academic communities and contributes to the progress and application of science with its publication.

Other selected journals from SCIRP are listed as below. Submit your manuscript to us via either submit@scirp.org or Online Submission Portal.
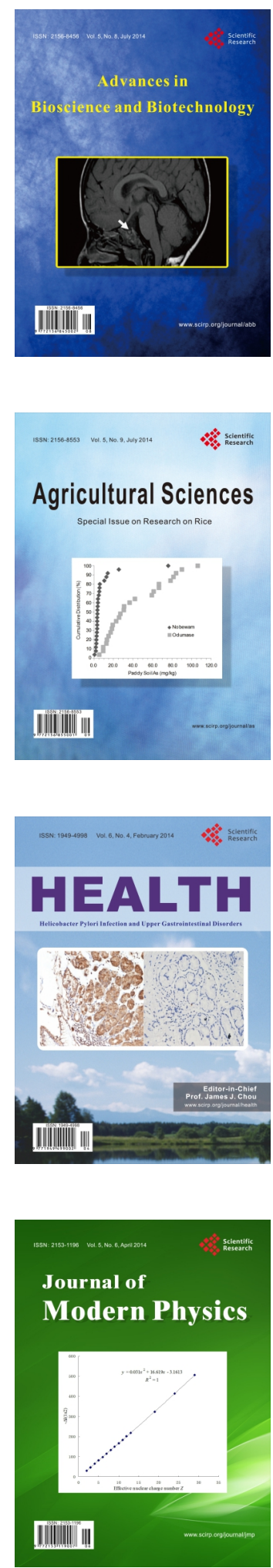
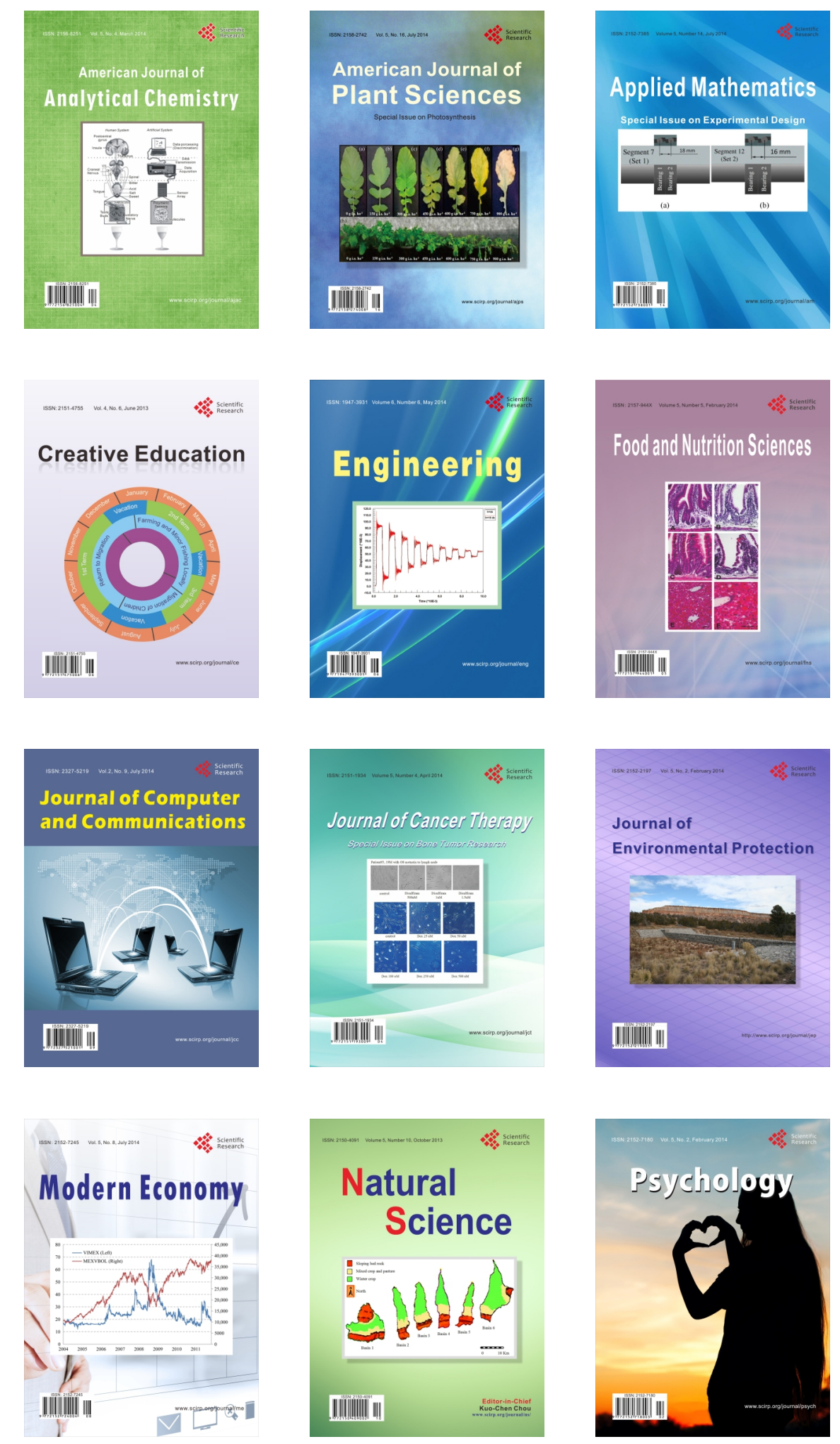